\title{
PRODUCT FORMULA FOR RESOLVENTS OF NORMAL OPERATORS AND THE MODIFIED FEYNMAN INTEGRAL
}

\author{
ANTÓNIO DE BIVAR-WEINHOLTZ AND MICHEL L. LAPIDUS
}

(Communicated by Palle E. T. Jorgensen)

\begin{abstract}
We extend the theory of the "modified Feynman integal" developed by the second author by extending his product formula for the imaginary resolvents of selfadjoint (unbounded) operators to those of normal operators. This enables us to establish the convergence of the "modified Feynman integral" for Hamiltonians with highly singular complex (instead of real) potentials. Such Hamiltonians arise naturally in the study of the Schrödinger equation associated with dissipative quantum mechanical systems.

By slightly altering the proof of our results, we also give a very general (operator-theoretic) interpretation of Nelson's "Feynman integral by analytic continuation in the mass parameter" that is valid for singular potentials with an arbitrary sign.

An interesting aspect of our "product formula for the imaginary resolvents of normal operators" is that it extends, and in some sense unifies, the above two approaches to the Feynman integral.
\end{abstract}

\section{INTRODUCTION}

In [L1, L2], a Trotter-like product formula was shown to converge to the unitary group generated by the generalized sum of nonnegative selfadjoint operators, using the imaginary resolvents of the intervening operators; in [L5], this result was generalized to the case where one of the operators has a negative part, relatively form bounded with respect to the other operator (with relative bound strictly less than one). This applies to a "modified Feynman integral" [L2-L7] when one specializes the intervening operators to be the usual $L^{2}$ Laplacian $-\Delta$ and the operator of multiplication by a real $L_{\text {loc }}^{1}$ function $q$, with some

Received by the editors September 9, 1989; this paper was presented by the first author to the Encontro da Sociedade Portuguesa de Matema'tica, Oporto, Portugal, April 1989 and by the second author to the Annual Meeting of the American Mathematical Society, Louisville, KY, January 1990.

1980 Mathematics Subject Classification (1985 Revision). Primary 47D05, 81C12; Secondary 28C20, 35J10, 47B15, 47B44, 47D45, 81C35.

Key words and phrases. Semigroups of operators, product formula for normal operators, modified Feynman integral, Schrödinger equation, Hamiltonians with singular complex potentials, dissipative quantum mechanical systems, magnetic vector potentials, analytic continuation in mass, Feynman path integrals, potentials with arbitrary signed singularity.

The work of the first author was partially supported by the J.N.I.C.T. under contract 87568 .

The work of the second author was partially supported by the NSF under grants DMS-8703138 and DMS-8904389. 
supplementary condition on its negative part (cf. [L5, §4]); in this manner, the second author has established in [L5] the existence of the "modified Feynman integral" (which he had introduced in [L2]) in the most general case for which the Schrödinger equation (with a real potential) can be solved without ambiguity.

In the present paper, we first show how the abstract formula can be extended to normal operators with sign conditions on their real and imaginary parts and relative form-boundedness of the negative real part of one of the operators with respect to the real part of the other. In the second and third sections, we consider the application to Schrödinger operators (hence, in particular, to a modified Feynman integral) in two distinct situations not contained in previously known results:

(1) The case of the Schrödinger equation with a highly singular complex potential (a magnetic vector potential is also allowed), which corresponds to the pseudo-Hamiltonians treated, for instance, in [E]. Formally, it is the operator

$$
\mathscr{H}=-(\vec{\nabla}-i \vec{a})^{2}+V \text {, }
$$

where $\vec{a} \in L_{\text {loc }}^{2}(\Omega ; \mathbb{R}), V=q-i q^{\prime}=q^{+}-q^{-}-i q^{\prime}$, and $q^{+}, q^{-}, q^{\prime}$ are real nonnegative functions in $L_{\text {loc }}^{1}(\Omega), q^{-}$is relatively form bounded with respect to $-\Delta$, with relative bound less than 1 , and $\Omega$ is a nonempty arbitrary open subset of $\mathbb{R}^{N}$. The new point is that one can consider a highly singular imaginary part in the potential $V$; in general, however, due to the term $-i q^{\prime}$, $e^{-i t \mathscr{H}}$ is no longer a group but only a semigroup. Physically, this corresponds to the fact that a quantum mechanical system with (nonreal) complex potential is dissipative (or "open," in the sense of [E]), rather than conservative.

Just as in [L2-L5], one can then establish the existence of the "modified Feynman integral" for Schrödinger operators with a highly singular complex potential.

(2) The case of the semigroup $e^{-t \mathscr{P}^{\prime}}$ generated by an operator $\mathscr{H}^{\prime}$ corresponding to the formal expression

$$
-(\vec{\nabla}-i \vec{a})^{2}-p+i\left(q-i q^{\prime}\right),
$$

$p$ a nonnegative real function with some adequate relative form-boundedness and local $L^{p}$ conditions, $\vec{a}, q, q^{\prime}$ with the same hypotheses as in (1), but without any restriction on $q^{-}$other than $L_{\text {loc }}^{1}(\Omega)$ (or, alternatively, $q \in L_{\text {loc }}^{1+\varepsilon}(\Omega)$, $\varepsilon>0$, depending on the restrictions on $p$ ). In this case, the proof has to be slightly modified, as the abstract result does not apply directly. The semigroup corresponds to a "Schrödinger equation with imaginary mass" and potential $q+i\left(p-q^{\prime}\right)$. The application to the Feynman integral for a real mass can be obtained (at least when $p \equiv 0$ ) by analytic continuation in the "mass parameter," a device introduced by E. Nelson (cf. [N]).

The theorem in (2) partially generalizes Theorem 4.1 of [B2] (detailed in [B3]; see also [N, K, and BP] for related results); we note that the product formula 
considered here holds for a scalar potential with the hypotheses of [BK] in their full generality, and a vector potential with optimal restrictions, which was not the case in [B2, B3], where, nevertheless, other formulae were considered.

\section{The ABSTRACt Result}

Let $T$ be a (not necessarily bounded) normal operator in a (complex) Hilbert space $H$ with inner product $\langle\cdot, \cdot\rangle$ and norm $\|\cdot\|$ (i.e., $T$ is a densely defined closed operator on $H$ such that $\left.T^{*} T=T T^{*}\right)$. Then one can write

$$
T=T_{1}-i T_{2},
$$

with $T_{1}, T_{2}$ selfadjoint operators on $H$, where $T_{1}, T_{2}$ are respectively the real and minus the imaginary parts of $T$, which can be defined by using the operational calculus given by the spectral theorem for normal operators, with the functions $\mathfrak{R}(z)$ and $-\mathfrak{I}(z)$ defined on the spectrum $\sigma(T) \subset \mathbb{C}$. (For all the elementary facts about unbounded normal operators, see, e.g., [R, pp. 348355].) Since $D(T)=D\left(T^{*}\right)$, it easily follows that

$$
T^{*}=T_{1}+i T_{2} .
$$

If one supposes $T_{1}, T_{2}$ to be nonnegative, then $i T$ is seen to be $m$-accretive (i.e., iT generates a contraction semigroup on $H$ ); this follows from the obvious fact that $i T$ and $(i T)^{*}$ are both accretive (see, e.g., [P, Corollary 4.4, p. $15])$. We shall denote by

$$
Q(T)=D\left(|T|^{1 / 2}\right)=D\left(\sqrt{T_{1}}\right) \cap D\left(\sqrt{T_{2}}\right)
$$

the form domain of $T ;|T|^{1 / 2}$ and $\sqrt{T_{j}}(j=1,2)$ can also be defined by means of the spectral theorem.

If now $A=A_{1}-i A_{2}, B=B_{1}-i B_{2}$ are normal operators on $H$ with $A_{j}$, $B_{j}$ nonnegative $(j=1,2)$ and

$$
Q=Q(A) \cap Q(B) \text { dense in } H,
$$

one can define the form sum $A+B$ as the operator in $H$ associated with the sesquilinear form

$$
\begin{gathered}
s: Q \times Q \subset H \times H \longrightarrow \mathbb{C} \\
(u, v) \mapsto\left\langle\sqrt{A_{1}} u, \sqrt{A_{1}} v\right\rangle-i\left\langle\sqrt{A_{2}} u, \sqrt{A_{2}} v\right\rangle \\
+\left\langle\sqrt{B_{1}} u, \sqrt{B_{1}} v\right\rangle-i\left\langle\sqrt{B_{2}} u, \sqrt{B_{2}} v\right\rangle ;
\end{gathered}
$$

i.e., $u \in D(A \dot{+} B)$ iff $v \mapsto s(u, v)$ is continuous on $Q$ for the $H$-topology, $(A+B) u$ being the unique vector of $H$, given by the Riesz representation theorem, such that

$$
\langle(A \dot{+} B) u, v\rangle=s(u, v), \quad \forall v \in Q .
$$

$i(A+B)$ is obviously accretive, and using the Lax-Milgram lemma, one can easily show that it is in fact $m$-accretive. The same conclusions still hold if 
one replaces the hypothesis " $B_{1}$ nonnegative" by a weaker assumption on its negative part $B_{-}$. (Note that $B_{1}=B_{+}-B_{-}$, where $B_{+}, B_{-}$are respectively the positive and negative parts of $B$, given by the spectral theorem.) Namely, we shall assume, as in [L5], that $B_{-}$is relatively form bounded with respect to $A_{1}$, with relative bound $<1$, i.e.,

$$
Q\left(B_{-}\right) \supset Q\left(A_{1}\right),
$$

and there exist positive constants $\gamma<1$ and $\delta$ such that:

$$
\left\|\sqrt{B_{-}} u\right\|^{2} \leq \gamma\left\|\sqrt{A_{1}} u\right\|^{2}+\delta\|u\|^{2}, \quad \forall u \in D\left(A_{1}\right) .
$$

In this case, in the definition of the sesquilinear form $s$, one has of course to replace the term $\left\langle\sqrt{B_{1}} u, \sqrt{B_{1}} v\right\rangle$ by $\left\langle\sqrt{B_{+}} u, \sqrt{B_{+}} v\right\rangle-\left\langle\sqrt{B_{-}} u, \sqrt{B_{-}} v\right\rangle$.

We can now state the following abstract result, which extends from selfadjoint to normal operators the "product formula for imaginary resolvents" obtained in [L5, Theorem 1, p. 263].

Theorem 1. Under the above hypotheses,

$$
\left([I+i(t / n) A]^{-1}[I+i(t / n) B]^{-1}\right)^{n} u \underset{n}{\longrightarrow} e^{-i t(A+B)} u,
$$

for all $u \in H$, uniformly in $t$ on bounded subsets of $[0,+\infty)$.

Proof. The proof parallels that of Theorem 1 in [L5], and we shall only indicate the changes to be made in the argument. Fix $\lambda>0, v \in H$; for $t>0$, set

$$
U_{t}=[I+t(\lambda+i A)]^{-1}, \quad V_{t}=[I+i t B]^{-1}
$$

and

$$
W_{t, \lambda}=t\left[U_{t}^{-1}-V_{t}\right]^{-1}, \quad w_{t}=W_{t, \lambda} v .
$$

Let us show that $w_{t} \longrightarrow[\lambda+i C]^{-1} v$, as $t \rightarrow 0^{+}$, where $C=A \dot{+} B$. One has $w_{t} \in D(A) \subset Q(A) \subset Q\left(B_{-}\right)$, and, by definition,

$$
v=\lambda w_{t}+A_{2} w_{t}+i A_{1} w_{t}+t^{-1}\left(I-V_{t}\right) w_{t} .
$$

By using the spectral theorem for normal operators, one obtains

$$
t^{-1}\left(I-V_{t}\right)=R_{B_{t}}+I_{B_{t}^{\prime}}+i\left(I_{B_{t}^{+}}-I_{B_{t}^{-}}\right),
$$

where $R_{B_{t}}, I_{B_{t}^{\prime}}, I_{B_{t}^{+}}$, and $I_{B_{t}^{-}}$are nonnegative bounded selfadjoint operators given by

$$
R_{B_{t}}=t|B|^{2}\left[\left(I+t B_{2}\right)^{2}+t^{2} B_{1}^{2}\right]^{-1}, \quad I_{B_{t}^{\prime}}=B_{2}\left[\left(I+t B_{2}\right)^{2}+t^{2} B_{1}^{2}\right]^{-1}
$$

and

$$
I_{B_{t}^{ \pm}}=B_{ \pm}\left[\left(I+t B_{2}\right)^{2}+t^{2} B_{1}^{2}\right]^{-1}
$$

Taking the inner product of (2) against $w_{t}$, one then has easily

$$
\left\|w_{t}\right\| \leq \lambda^{-1}\|v\|
$$


hence, also $\left\|\sqrt{A_{2}} w_{t}\right\|,\left\|\sqrt{R_{B_{t}}} w_{t}\right\|$, and $\left\|\sqrt{I_{B_{t}^{\prime}}} w_{t}\right\|$ are bounded independently of $t$. This, along with the observation (made in [L5], just before Equation (12), p. 266) that

$$
\left\|\sqrt{I_{B_{t}^{-}}} \xi\right\| \leq\left\|\sqrt{B_{-}} \xi\right\|, \quad \forall \xi \in Q\left(B_{-}\right),
$$

(a consequence of the spectral theorem), allows us to apply hypothesis (1) to deduce, as in [L5, p. 266], that $\left\|\sqrt{I_{B_{t}^{ \pm}}} w_{t}\right\|$ and $\left\|\sqrt{A_{1}} w_{t}\right\|$ are also bounded independently of $t$. We can then pass to the limit, weakly in $H$, along a sequence $t_{n} \underset{n}{\longrightarrow} 0 ; w_{t}$ tends to a certain $w$, and to identify the limits of the other bounded sequences, one can use the spectral and Lebesgue theorems. We first obtain, in particular, that $w \in Q(A) \cap Q(B)=Q$. Note also that by the $m$-accretiveness of $i B$, one has

$$
\sigma(B) \subset\{z \in \mathbb{C}: \mathfrak{I}(z) \leq 0\} ;
$$

so that, for $z=x+i y \in \sigma(B)$, we obtain

$$
\begin{aligned}
t|z|^{2}\left[(1-t y)^{2}+t^{2} x^{2}\right]^{-1} & =\frac{t|z|}{\sqrt{(1-t y)^{2}+t^{2} x^{2}}} \frac{|z|}{\sqrt{(1-t y)^{2}+t^{2} x^{2}}} \\
& \leq \frac{t|z|}{\sqrt{t^{2}\left(x^{2}+y^{2}\right)}} \frac{|z|}{\sqrt{1}}=|z| .
\end{aligned}
$$

Hence, in fact,

$$
\left\|\sqrt{R_{B_{t}}} \xi\right\| \underset{t \rightarrow 0^{+}}{\longrightarrow} 0, \quad \forall \xi \in Q(B)
$$

from which one easily deduces now that, along $t_{n}$,

$$
\sqrt{R_{B_{t}}} w_{t} \longrightarrow 0, \text { weakly. }
$$

Now, let $y \in Q=Q(A) \cap Q(B)$; the inner product of (2) with $y$ gives, upon passing to the limit in the second member,

$$
\begin{aligned}
\langle v, y\rangle= & \lambda\left\langle w_{t}, y\right\rangle+\left\langle\sqrt{A_{2}} w_{t}, \sqrt{A_{2} y}\right\rangle+\left\langle\sqrt{R_{B_{t}}} w_{t}, \sqrt{R_{B_{t}} y}\right\rangle+\left\langle\sqrt{I_{B_{t}^{\prime}}} w_{t}, \sqrt{I_{B_{t}^{\prime}} y}\right\rangle \\
& +i\left[\left\langle\sqrt{A_{1}} w_{t}, \sqrt{A_{1}} y\right\rangle+\left\langle\sqrt{I_{B_{t}^{+}}} w_{t}, \sqrt{I_{B_{t}^{+}}} y\right\rangle-\left\langle\sqrt{I_{B_{t}^{-}}} w_{t}, \sqrt{I_{B_{t}^{-}} y}\right\rangle\right] \\
= & \lambda\langle w, y\rangle+i s(w, y) ;
\end{aligned}
$$

so that, by definition of $C=A \dot{+} B, w \in D(C)$, and

$$
(\lambda+i C) w=v
$$

i.e., $w_{t}$ tends weakly to $w=(\lambda+i C)^{-1} v$.

The remainder of the proof can be carried out just as in [L5, pp. 267-268], and we omit it.

Remark 1. (a) A corollary analogous to [L5, Corollary 1, p. 269]-in which the form domain $Q(C)$ is not necessarily densely defined in $\mathrm{H}$-is also available. 
(b) Even in the case of nonnegative selfadjoint operators [L1, L2], let alone in the case studied in [L5] or the present situation of normal operators, it is not known whether one can replace the "imaginary resolvents" by the corresponding semigroups in the statement of Theorem 1. See [L2-L7], especially [L2], for a discussion of this very difficult and intriguing problem.

\section{Application to a modified Feynman integral}

We specialize now to $H=L^{2}(\Omega), \Omega$ a nonempty open subset of $\mathbb{R}^{N}$, $N \geq 1$. Let $A=-\Delta$ be the nonnegative Laplacian in $H$, and $B$ the maximal multiplication operator by a function $V=q^{+}-q^{-}-i q^{\prime}$, where $q^{+}, q^{-}, q^{\prime}$ are real nonnegative functions in $L_{\text {loc }}^{1}(\Omega), q^{-}$relatively form bounded with respect to $-\Delta$, with relative bound less than 1 . In this case, the form sum $A \dot{+} B$ extends, hence coincides with, the natural realization $\mathscr{H}$ of $-\Delta+V(x)$, with domain

$$
D(\mathscr{H})=\left\{u \in H_{0}^{1}(\Omega): V u \in L_{\text {loc }}^{1}(\Omega),-\Delta u+V u \in H\right\} .
$$

In fact, $\mathscr{H}+\lambda$ is known to be $m$-accretive for some $\lambda>0$ (see, e.g., [BK]). In the case $\Omega=\mathbb{R}^{N}$, with some supplementary assumptions on $q^{-}$, one can even replace " $u \in H_{0}^{1}(\Omega)$ " by " $u \in L^{2}(\Omega)$ " in the definition of the domain (see [BK]). (The hypothesis $q^{\prime} \geq 0$ is not required for the results of [BK] to hold and a somewhat stronger hypothesis is assumed on $q^{-}$, but then it is the closure of the operator $\mathscr{H}+\lambda$ that is known to be $m$-accretive. In the case when $q^{\prime}$ has a sign, however, even with the stated hypothesis on $q^{-}$, the methods of [BK] easily adapt to show that $\mathscr{H}+\lambda$ itself is $m$-accretive with $D(\mathscr{H}) \subset D(A \dot{+} B)$.)

We can then apply Theorem 1 to $A$ and $B$, which gives us a representation of the solutions of the Schrödinger equation

$$
i \frac{\partial \Psi}{\partial t}=\mathscr{H} \Psi, \quad(t \geq 0)
$$

corresponding to the pseudo-Hamiltonian $\mathscr{H}$. (See [E] for a description of the properties and physical relevance of such operators to the study of "open", i.e., nonisolated, quantum systems.) When $\Omega=\mathbb{R}^{N}$, this gives us an explicit representation of $e^{-i t \mathscr{H}}$ as a limit of iterated integral operators that generalizes the modified Feynman path integral introduced by the second author [L2-L7] to the case of a complex potential with a highly singular (merely $L_{\text {loc }}^{1}$ ) negative imaginary part. (See [L2, §6.B], as well as [L5, §4, in particular Theorem 2, p. 270], and [L6, §4c] or [L7, Part I] for the definition and properties of the modified Feynman integral in the case of real potentials.)

We can also replace $-\Delta$ by a more general elliptic operator $L$ with a vector potential, formally,

$$
L=L(\vec{a})=-\sum_{j, k=1}^{N}\left(\partial_{k}-i a_{k}\right)\left[b_{j k}\left(\partial_{j}-i a_{j}\right)\right],
$$


where the $b_{j k}$ form a symmetric uniformly elliptic matrix of real-bounded measurable functions on $\Omega$ and $\vec{a}$ is an $N$-dimensional vector of $L_{\text {loc }}^{2}$ real functions on $\Omega$. $L$ is the nonnegative selfadjoint operator associated with the closure of the minimal energy form corresponding to (4) (i.e., the closure $h$ of the form defined on $\left.C_{0}^{\infty}(\Omega)\right)$. The form domain of $L$ is

$$
Q(h)=Q(L)=\left\{u \in L^{2}(\Omega):|u| \in H_{0}^{1}(\Omega), \vec{\nabla} u-i \vec{a} u \in L^{2}(\Omega)\right\},
$$

and $L$ is the maximal restriction, as an operator on $H$, of the operator

$$
\begin{gathered}
\tilde{L}: Q(h) \longrightarrow Q(h)^{\prime} \subset \mathscr{D}^{\prime}(\Omega) \\
u \mapsto \widetilde{L} u=h(u, \overline{)})=-\sum_{j, k=1}^{N} \int_{\Omega} b_{j k}\left[\left(\partial_{j}-i a_{j}\right) u\right]\left[\left(\partial_{k}-i a_{k}\right) \cdot\right] d x .
\end{gathered}
$$

See [B1-B3] for the definition of such operators and for the properties that enable us to apply Theorem 1 in this case.

Remark 2. It would be interesting to extend to normal operators the abstract perturbation theorem established in [L6, Theorem 3.1, p. 43] in order to extend to singular complex potentials the "dominated convergence theorem" for Feynman integrals obtained in [L6, Theorem 4.1, p. 52, and Corollary 4.1, p. 57].

\section{THE CASE OF IMAGINARY MASS}

With the notation of the preceding section, we can also take $A=-i A_{2}=i \Delta$, and $B$ as in $\S 2$. If one wanted to apply Theorem 1 directly, hypothesis (1) would impose that $q^{-}$is essentially bounded, as now $A_{1}=0$. In this case, however, we can modify the proof of Theorem 1 to obtain the analogous result by assuming only that $q^{-} \in L_{\mathrm{loc}}^{1}(\Omega)$; i.e., the real part of the potential can be any locally summable function on $\Omega$. We can also add a nonnegative imaginary part $p$ to the potential with some boundedness hypothesis relatively to $-\Delta$, provided that, in the product formula, $p$ appears in the same resolvent factor as $-\Delta$. (Otherwise, the resolvent associated with the potential would, in general, be unbounded.) Finally, as above, we can replace $-\Delta$ by the general operator $L$. Under these general hypotheses, one can no longer define " $A+B$ " as a form sum in a suitable way, but we can consider an m-accretive realization of " $i(A+B)$ ", defined in [BK] for the case $A=i \Delta$ and in [B1, B3], for $A=-i L$. We shall consider the following set of hypotheses, exactly the same as in [BK]:

$$
V \in L_{\mathrm{loc}}^{1}(\Omega), \quad V=q+i\left(p-q^{\prime}\right),
$$

$$
q, p, q^{\prime} \text { real-valued functions, } \quad p, q^{\prime} \geq 0 \text {, }
$$

$$
p \in L^{\infty}(\Omega)+L^{p}(\Omega), \quad \text { with } \begin{cases}p=\frac{N}{2} & \text { when } N \geq 3 \\ p>1 & \text { when } N=2 \\ p=1 & \text { when } N=1,\end{cases}
$$


and

$$
\text { either } q \in L_{\mathrm{loc}}^{1+\varepsilon}(\Omega) \text { or } p \in L_{\mathrm{loc}}^{(N / 2)+\varepsilon}(\Omega) \text {, }
$$

when $N \geq 2, \quad$ for some arbitrarily small $\varepsilon>0$.

We note that hypothesis (5.iii) can be replaced by the weaker assumption that $p \in L_{\text {loc }}^{1}(\Omega)$ is infinitesimally form bounded with respect to $-\Delta$, i.e., $\forall \delta>0$, there exists $\lambda_{\delta}>0$ such that

$$
\int_{\Omega} p|u|^{2} \leq \delta \int_{\Omega}|\vec{\nabla} u|^{2}+\lambda_{\delta} \int_{\Omega}|u|^{2}, \quad \forall u \in H_{0}^{1} .
$$

Since

$$
|\vec{\nabla}| u|| \leq|(\vec{\nabla}-i \vec{a}) u|, \quad \forall u \in Q(L),
$$

(cf. [LS] or [B3]), this hypothesis implies, in particular, that we can define the form sum $L \dot{+}\left(-p+\lambda_{\alpha}\right)=L \dot{-} p+\lambda_{\alpha}$ as a positive selfadjoint operator with form domain $Q(L), \alpha$ being the ellipticity constant of $\left[b_{j k}\right], \lambda_{\alpha}$ given by (5.v). $\left(L \dot{-} p+\lambda_{\alpha}\right.$ is the maximal restriction to an operator on $H$ of

$$
\widetilde{L}-p+\lambda_{\alpha} \in \mathscr{L}\left(Q(L), Q(L)^{\prime}\right),
$$

see [B3, II, §2].)

Then it is known that the operator $i C$ such that

$$
\begin{gathered}
D(C)=\left\{u \in Q(L): V u \in L_{\mathrm{loc}}^{1}(\Omega), \widetilde{L} u+i V u \in H\right\}, \\
C u=-i \widetilde{L} u+V u, \quad \forall u \in D(C),
\end{gathered}
$$

is closable, its closure $i \bar{C}$ being such that $i \bar{C}+\lambda_{\alpha}$ is $m$-accretive. See [BK] for the case $L=-\Delta$ and [B3] for the general case. In the adaptation of the proof in $[\mathrm{BK}$ ] to a general $L$, the following lemma plays a crucial role and it will also be used later.

Lemma 1. Under the above hypotheses, let $g \in L^{\infty}(\Omega)$ with a nonnegative real part, and let $u \in H$; then, for $\lambda>\lambda_{\alpha}$ :

$$
\left|(L(\vec{a}) \dot{-} p+g+\lambda)^{-1} u\right| \leq(L(\overrightarrow{0}) \dot{-} p+\lambda)^{-1}|u|, \quad \text { a.e. in } \Omega \text {. }
$$

Proof of Lemma 1. This lemma is closely related to Lemma 6 of [LS], whose proof can be easily adapted to this situation (see [B3, Lemma 2.1, p. 28]). We just note that in [LS], although $g$ is taken nonnegative and $p \equiv 0, g$ is only supposed $L_{\mathrm{loc}}^{1}$. Since we suppose that $g \in L^{\infty}(\Omega)$, we do not need to start with $u$ in $L^{\infty}$ and the proof does not require the use of Lemma 4 in [LS].

The solution of the general Schrödinger equation with imaginary mass and complex potential $V$ :

$$
i \frac{\partial \Psi}{\partial t}=(-i L(\vec{a})+V) \Psi, \quad(t \geq 0)
$$

is given by the semigroup $e^{-i t \bar{C}}$, for which one has the following product formula. 
Theorem 2. Under the above hypotheses,

$$
\left(\left[I+(t / n)\left(L \dot{-} p+\lambda_{\alpha}\right)\right]^{-1}\left[I+i(t / n)\left(q-i q^{\prime}\right)\right]^{-1}\right)^{n} u \underset{n}{\longrightarrow} e^{-i t\left(\bar{C}-i \lambda_{\alpha}\right)} u,
$$

for all $u \in H$, uniformly in $t$ on bounded subsets of $[0,+\infty)$.

Proof of Theorem 2. We shall use the notation of the proof of Theorem 1, with

$$
A=-i A_{2}=-i\left(L \dot{-} p+\lambda_{\alpha}\right), \quad B=q-i q^{\prime} .
$$

We now take $v \in L^{2}(\Omega) \cap L^{\infty}(\Omega)$, and we first show that:

$$
w_{t} \rightarrow\left[\lambda+i\left(C-i \lambda_{\alpha}\right)\right]^{-1} v,
$$

along a sequence $t_{n} \underset{n}{\rightarrow}$, weakly in $H$.

One has

$$
w_{t} \in D(A)=D(L \dot{-} p) \subset Q(L),
$$

but now $w_{t}$ is the solution of the following elliptic equation:

$$
v=\left(L \dot{-} p+\lambda_{\alpha}\right) w_{t}+\lambda w_{t}+R_{t} w_{t}+I_{q_{t}^{\prime}} w_{t}+i I_{q_{t}} w_{t},
$$

with

$$
R_{t}=t\left(q^{2}+q^{\prime 2}\right)\left[\left(I+t q^{\prime}\right)^{2}+t^{2} q^{2}\right]^{-1}, \quad I_{q_{t}^{\prime}}=q^{\prime}\left[\left(I+t q^{\prime}\right)^{2}+t^{2} q^{2}\right]^{-1}
$$

and

$$
I_{q_{t}}=q\left[\left(I+t q^{\prime}\right)^{2}+t^{2} q^{2}\right]^{-1}
$$

As in the proof of Theorem 1, one can easily bound

$$
\left\|w_{t}\right\|, \quad\left\|\sqrt{L \dot{-} p+\lambda_{\alpha}} w_{t}\right\|, \quad\left\|\sqrt{R_{t}} w_{t}\right\|, \quad \text { and } \quad\left\|\sqrt{I_{q_{t}^{\prime}}} w_{t}\right\|
$$

independently of $t$; and so we can justify that for a sequence $t_{n} \underset{n}{\rightarrow} 0, w_{t}$ converges weakly in $H$ to

$$
w \in Q\left(A_{2}\right)=Q(L)
$$

with

$$
q^{\prime}|w|^{2} \in L^{1}(\Omega)
$$

In particular, $q^{\prime} w \in L_{\text {loc }}^{1}(\Omega)$. If we multiply (6) by a test function

$$
\varphi \in C_{0}^{\infty}(\Omega) \subset Q\left(A_{2}\right) \cap L^{2}\left(\sqrt{q^{\prime}} d x\right) \cap L^{2}\left(\sqrt{q^{2}+q^{\prime 2}} d x\right),
$$

and integrate on $\Omega$, we can pass to the limit in the right-hand side of the equation obtained in this fashion, much as in the proof of Theorem 1. We point out the differences in the argument. For the term in $R_{t}$, one has to use

$$
\left\|\sqrt{R_{B_{t}}} \varphi\right\|_{t \rightarrow 0^{+}}^{\rightarrow} 0, \quad \forall \varphi \in C_{0}^{\infty}(\Omega),
$$


a consequence of Lebesgue theorem, and estimate (3), combined with the hypothesis $V \in L_{\text {loc }}^{1}(\Omega)$. For the last term,

$$
\int_{\Omega} i I_{q_{t}} w_{t} \varphi
$$

we notice that, by the Lebesgue theorem,

$$
I_{q_{t}} \varphi \underset{t \rightarrow 0^{+}}{\rightarrow} q \varphi
$$

either in $L^{1+\varepsilon}(\Omega)$, or in $L^{1}(\Omega)$, according to the hypotheses on $q$ (cf. (5.iv)). To pass to the limit in this term, it is then sufficient to obtain a bound on $w_{t}$ either in $L^{(1+\varepsilon)^{\prime}}(\operatorname{supp} \varphi)$ or in $L^{\infty}(\operatorname{supp} \varphi)$, independently of $t$. This can be achieved by using Lemma 1 with $g:=R_{t}+I_{q_{t}^{\prime}}+i I_{q_{t}}$.

Combining with $(6)$, one obtains:

$$
\left|w_{t}\right| \leq \psi, \quad \text { a.e. in } \Omega,
$$

where $\psi$ is the unique $H_{0}^{1}(\Omega)$ solution of

$$
\widetilde{L}(\overrightarrow{0}) \psi-p \psi+\left(\lambda+\lambda_{\alpha}\right) \psi=|v| .
$$

But now, hypothesis (5.v) and an easy generalization of Theorem 2.3 of [BK] ensure that

$$
\psi \in \bigcap_{2 \leq p<\infty} L^{p}(\Omega) .
$$

Hence, in particular, $w_{t}$ is uniformly bounded in $L^{(1+\varepsilon)^{\prime}}(\Omega)$, which allows to conclude in the case $q \in L_{\text {loc }}^{1+\varepsilon}(\Omega)$.

In the alternative hypothesis $p \in L_{\text {loc }}^{(N / 2)+\varepsilon}(\Omega)$, we can apply standard elliptic local regularity to equation (10) to conclude that $\psi \in L_{\text {loc }}^{\infty}(\Omega)$. Hence $w_{t}$ is uniformly bounded in $L^{\infty}(\operatorname{supp} \varphi)$; so, once again we can pass to the limit in (9), which guarantees, $\varphi \in C_{0}^{\infty}(\Omega)$ being arbitrary, that

$$
q w \in L_{\text {loc }}^{1}(\Omega) \text {. }
$$

From (5.v), (8), and (11), we deduce that

$$
V w \in L_{\text {loc }}^{1}(\Omega) \text {. }
$$

We can then pass to the limit in (6) in the sense of distributions, which gives

$$
\begin{aligned}
\int_{\Omega} v \varphi & =\left\langle\sqrt{L \dot{-} p+\lambda_{\alpha}} w, \sqrt{L \dot{-} p+\lambda_{\alpha}} \bar{\varphi}\right\rangle+\int_{\Omega}\left(\lambda+q^{\prime}+i q\right) w \varphi \\
& =h(w, \bar{\varphi})-\int_{\Omega} p w \varphi+\lambda_{\alpha} \int_{\Omega} w \varphi+\int_{\Omega}\left(\lambda+q^{\prime}+i q\right) w \varphi
\end{aligned}
$$

$\forall \varphi \in C_{0}^{\infty}(\Omega)$, and by definition of $\widetilde{L}$, we obtain from (13), in $\mathscr{D}^{\prime}(\Omega)$ :

$$
v=\tilde{L} w-p w+\lambda_{\alpha} w+\left(\lambda+q^{\prime}+i q\right) w=\tilde{L} w+i V w+\left(\lambda+\lambda_{\alpha}\right) w
$$


We thus have, in particular,

$$
\tilde{L} w+i V w=v-\left(\lambda+\lambda_{\alpha}\right) w \in H
$$

(12) and (14) now imply

$$
w \in D(C), \quad\left[\lambda+i\left(C-i \lambda_{\alpha}\right)\right] w=v .
$$

We have concluded that along a sequence $t_{n}$ tending to $0^{+}, w_{t}$ tends weakly to

$$
w=\left[\lambda+i\left(\bar{C}-i \lambda_{\alpha}\right)\right]^{-1} v
$$

Using the same argument as in the proof of Theorem 1 of [L5], we can easily conclude that we have strong convergence as $t \rightarrow 0^{+}$. The density of $L^{2}(\Omega) \cap$ $L^{\infty}(\Omega)$ in $L^{2}(\Omega)$, and the boundedness of the operators involved, now allow us to reach the same conclusion for a general $v \in L^{2}(\Omega)$. The remainder of the proof can be performed exactly as in [L5].

Remark 3. (a) The above results in $\S 3$ allow more general conditions on the complex (time independent) potential than those allowed by probabilistic methods about the "analytic operator-valued Feynman integral." (See e.g. [J, §3] and the references therein, especially $[\mathrm{C}, \mathrm{N}$, and $\mathrm{H}]$.)

(b) Assume that $\Omega=\mathbb{R}^{N} \backslash F$ as in $[N]$, where $F$ is a closed set of capacity zero. Then, as was noted in [K, Remark 2.5 and Lemma 2.6, p. 107], we have $L^{2}(\Omega)=L^{2}\left(\mathbb{R}^{N}\right)$ and $H_{0}^{1}(\Omega)=H^{1}\left(\mathbb{R}^{N}\right)$. Hence, the results of $\S 3$ can be applied to potentials that are arbitrarily singular on $F$ (and, in particular, are more general than those in $[\mathrm{N}],[\mathrm{H}]$, or $[\mathrm{J}])$.

\section{REFERENCES}

[BP] A. Bivar-Weinholtz and R. Piraux, Formule de Trotter pour l'opérateur $-\Delta+q^{+}-q^{-}+i q^{\prime}$, Ann. Fac. Sci. Toulouse Math. 5 (1983), 15-37.

[B1] A. Bivar-Weinholtz, Sur l'opérateur de Schrödinger avec potentiel singulier magnétique, dans un ouvert arbitraire de $\mathbb{R}^{N}$, C. R. Acad. Sci. Paris Sér. A 294 (1982), 213-216.

[B2] _ Opérateurs de Schrödinger avec potentiel singulier magnétique, dans un ouvert arbitraire de $\mathbb{R}^{N}$, Portugal Math. 41 (1982), 1-12.

[B3] __ Operadores de Schrödinger com potenciais singulares, Ph.D. dissertation, University of Lisbon, Portugal, 1982.

[BK] H. Brézis and T. Kato, Remarks on the Schrödinger operator with singular complex potentials, J. Math. Pures Appl. 58 (1979), 137-151.

[C] R. H. Cameron, A family of integrals serving to connect the Wiener and Feynman integrals, J. Math. and Phys. Sci. 39 (1960), 126-140.

[E] P. Exner, Open quantum systems and Feynman integrals, Fund. Theories Physics, Reidel, Dordrecht, Boston, MA, 1985.

[H] B. O. Haugsby, An operator valued integral in a function space of continuous vector valued functions, Ph.D. dissertation, University of Minnesota, Minneapolis, MN, 1972.

[J] G. W. Johnson, Existence theorems for the analytic operator-valued Feynman integral, Sém. Anal. Moderne, 1988, no. 20, Publications Mathématiques, Université de Sherbrooke (J. Dubois and P. Morales, eds.), Québec, Canada, 1988. 
[K] T. Kato, On some Schrödinger operators with a singular complex potential, Ann. Scuola Norm. Sup. Pisa Cl. Sci. (4) 5 (1978), 105-114.

[L1] M. L. Lapidus, Formules de moyenne et de produit pour les résolvantes imaginaires d'opérateurs auto-adjoints, C. R. Acad. Sci. Paris Sér. A 291 (1980), 451-454.

[L2] _ The problem of the Trotter-Lie formula for unitary groups of operators, Séminaire Choquet: Initiation à l'Analyse, Publ. Math. Université Pierre et Marie Curie 46, 1980-81, 20ème année (1982), 1701-1745.

[L3] _ Modification de l'intégrale de Feynman pour un potentiel positif singulier: approche séquentielle, C. R. Acad. Sci. Paris Sér. A 295 (1982), 1-3.

[L4] __ Intégrale de Feynman modifiée et formule de produit pour un potentiel singulier négatif, C. R. Acad. Sci. Paris Sér. A 295 (1982), 719-722. Product formula, imaginary resolvents and modified Feynman integral, in Proc. Symp. Pure Math. (F. E. Browder, ed.), Vol. 45, Part 2, Amer. Math. Soc., Providence, RI, 1986, pp. 109-112.

[L5] _ Product formula for imaginary resolvents with application to a modified Feynman integral, J. Funct. Anal. 63 (1985), 261-275.

[L6] _ Perturbation theory and a dominated convergence theorem for Feynman integrals, Integral Equations Operator Theory 8 (1985), 36-62.

[L7] _ Formules de Trotter et calcul opérationnel de Feynman, Thèse de Doctorat d'Etat ès Sciences, Mathématiques, Université Pierre et Marie Curie (Paris VI), France, June 1986. (Part I: Formules de Trotter et intégrales de Feynman)

[LS] H. Leinfelder and C. G. Simader, Schrödinger operators with singular magnetic vector potentials, Math. Z. 176 (1981), 1-19.

[N] E. Nelson, Feynman integrals and the Schrödinger equation, J. Math. Phys. 5 (1964), 332343.

[P] A. Pazy, Semigroups of linear operators and applications to partial differential equations, Springer-Verlag, New York, 1983.

[R] W. Rudin, Functional analysis, Tata McGraw-Hill, New Delhi, 1974.

C.M.A.F., 2 Avenue de Gama Pinto, 1699 Lisbon Codex, Portugal

Department of Mathematics, Boyd Graduate Studies Research Center, University of Georgia, Athens, Georgia 30602

Current address, Michel L. Lapidus: Department of Mathematics and Computer Science, University of California, Riverside, California 92521-0135 